\title{
Erratum to: Amelioration of 2,4,6-trinitrobenzene sulfonic acid-induced colitis in mice by immunoregulatory dendritic cells
}

\author{
Shoichi Hoshino $\cdot$ Akiko Kurishima $\cdot$ Muneo Inaba $\cdot$ Yugo Ando $\cdot$ Toshiro Fukui • \\ Kazushige Uchida • Akiyoshi Nishio • Hiroshi Iwai - Takashi Yokoi - Tomoki Ito • \\ Sanae Hasegawa-Ishii $\cdot$ Atsuyoshi Shimada $\cdot$ Ming Li $\cdot$ Kazuichi Okazaki $\cdot$ Susumu Ikehara
}

Published online: 11 August 2012

(C) Springer 2012

Erratum to: J Gastroenterol (2011) 46:1368-1381
DOI 10.1007/s00535-011-0460-4

In the above-cited article, incorrect versions of Figs. 5a and 8 a were published. The correct figures appear below.

The online version of the original article can be found under doi:10.1007/s00535-011-0460-4.

S. Hoshino $\cdot$ A. Kurishima $\cdot$ Y. Ando $\cdot$ T. Fukui $\cdot$ K. Uchida $\cdot$ A. Nishio $\cdot$ K. Okazaki

The Third Department of Internal Medicine,

Division of Gastroenterology and Hepatology,

Kansai Medical University, Moriguchi, Osaka, Japan

e-mail: hoshinos@takii.kmu.ac.jp

A. Kurishima

e-mail: kurishia@takii.kmu.ac.jp

T. Fukui

e-mail: fukuitos@takii.kmu.ac.jp

K. Uchida

e-mail: uchidak@takii.kmu.ac.jp

A. Nishio

e-mail: nishioa@takii.kmu.ac.jp

K. Okazaki

e-mail: okazaki@hirakata.kmu.ac.jp

M. Inaba

First Department of Pathology, Kansai Medical University,

Moriguchi, Osaka, Japan

e-mail: inabam@takii.kmu.ac.jp

H. Iwai

Department of Otolaryngology, Kansai Medical University,

Moriguchi, Osaka, Japan

e-mail: iwai@takii.kmu.ac.jp
T. Yokoi $\cdot$ T. Ito

First Department of Internal Medicine,

Kansai Medical University, Moriguchi, Osaka, Japan

e-mail: yokoit@hirakata.kmu.ac.jp

T. Ito

e-mail: itot@takii.kmu.ac.jp

S. Hasegawa-Ishii · A. Shimada

Department of Pathology, Institute for Developmental Research,

Aichi Human Service Center, Kasugai, Aichi, Japan

e-mail: sanae@inst-hsc.jp

A. Shimada

e-mail: ats7@ma.ccnw.ne.jp

M. Li · S. Ikehara $(\bowtie)$

Department of Stem Cell Disorders, Kansai Medical University, 10-15 Fumizono-cho, Moriguchi, Osaka 570-8506, Japan e-mail: ikehara@takii.kmu.ac.jp

M. Li

e-mail: liming@takii.kmu.ac.jp 


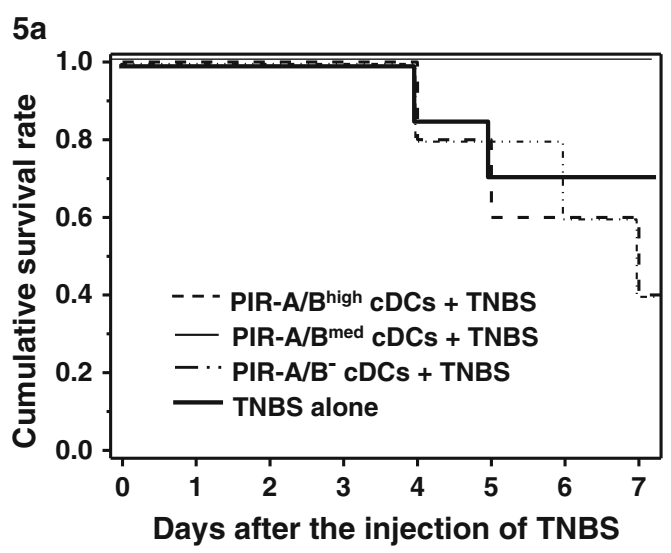

$8 a$

TNBS alone
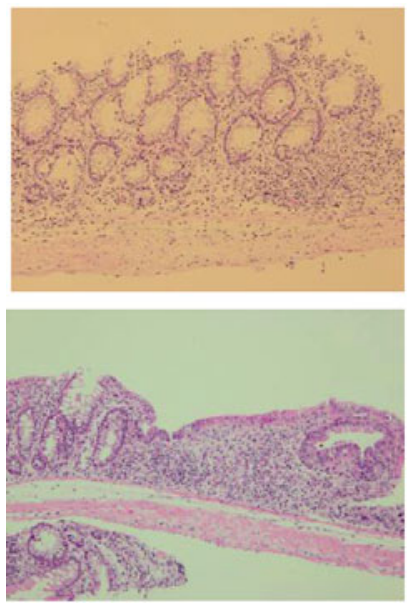

TNBS + PIR ${ }^{\text {med }}$ cDCs

(treated with 1-mT)
TNBS + PIR ${ }^{\text {med }}$ cDCs
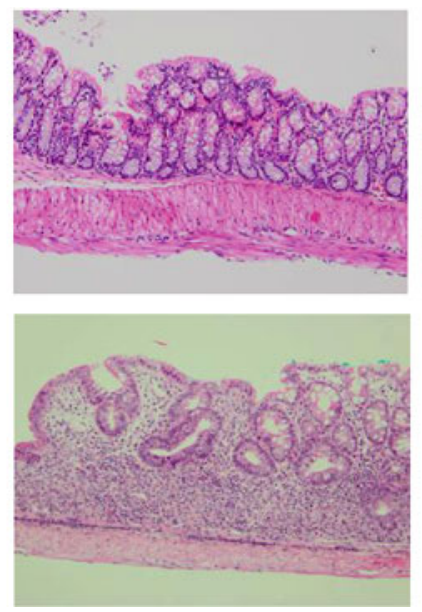

TNBS + 1-mT
TNBS + PIR ${ }^{\text {med }} \mathrm{cDCs}+1-\mathrm{mT}$

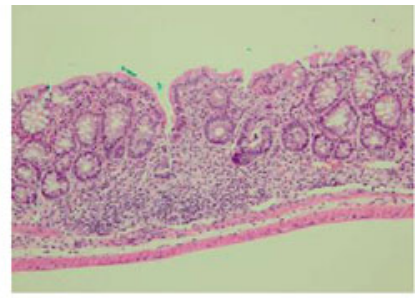

